05,12

\title{
Движение взаимодействующих магнитных вихрей В параллельных нанолентах
}

\author{
(C) В.А. Орлов ${ }^{1,2}$, А.А. Иванов ${ }^{1}$, И.Н. Орлова ${ }^{3}$ \\ ${ }^{1}$ Сибирский фредеральный университет, \\ Красноярск, Россия, \\ ${ }^{2}$ Институт физики им. Л.В. Киренского ФИЦ КНЦ СО РАН, \\ Красноярск, Россия \\ ${ }^{3}$ Красноярский государственный педагогический университет им. В.П. Астафьева, \\ Красноярск, Россия \\ E-mail: orlhome@rambler.ru
}

(Поступила в Редакцию 18 октября 2018 г.)

\begin{abstract}
Теоретически исследуется периодическое движение взаимодействующих вихревых доменных стенок в паре нанолент. В качестве модели рассмотрены две параллельно расположенные наноленты с неоднородностями намагниченности в виде магнитных вихрей. Магнитные подсистемы лент взаимодействуют магнитостатически. Квазиупругая связь между вихрями обеспечивает существование нормальных мод периодического движения намагниченности. Вычислены частоты этих мод. Показано, что не любые сочетания топологического заряда вихрей приводят к резонансному поведению намагниченности в переменных полях. Обсуждается влияние статической составляющей магнитного поля на частоту периодического движения вихревых доменных стенок.
\end{abstract}

Исследование выполнено при финансовой поддержке РФФИ в рамках научного проекта № 18-02-00161.

DOI: $10.21883 /$ FTT.2019.03.47241.285

\section{1. Введение}

Внимание к низкомерным объектам, таким как нанопроволоки и наноленты связано не только с перспективами конструирования на их основе разнообразных устройств спинтроники [1-3], но и с возможностью разрешения многих фундаментальных проблем магнетизма. Особенно пристально исследуются процессы перемагничивания. Вместе с тем, аналитическое описание магнитных свойств нанолент сопряжено с существенными вычислительными трудностями, что связано со сложной структурой намагниченности и особенностями поведения в переменных полях. Поэтому особый интерес вызывают процессы эволюции доменной структуры нанолент под действием переменного магнитного поля.

В зависимости от геометрии лент (соотношения линейных размеров) и их магнитных характеристик реализуются различные виды доменных стенок: традиционные - типа Нееля (transverse wall (TW)), вихревые (vortex wall $(\mathrm{VW})$ ) и их сложные комбинации [4-11]. Разные типы DW в лентах интенсивно изучаются теоретически (например $[10,12])$ и экспериментально (например $[13,14])$. Естественно, что характер движения стенок со столь сложными конфигурациями содержит необычные эффекты и вызывает интерес исследователей. В частности, были обнаружены и сейчас уже достаточно хорошо изучены несколько режимов движения стенок с вихревой структурой под действием статического поля (или спин-поляризованного тока) в зависимости от его величины [15-20]. Интересно, что наличие вихревой структуры обуславливает существование циклического движения стенки со смещением - дрейфа.

В массивах близко расположенных проволок (лент) нельзя исключать взаимное влияние их магнитных подсистем. Взаимодействие топологических неоднородностей намагниченности является существенным и влияет на процессы перемагничивания [21,22]. В настоящей работе мы ставим целью описание циклического характера движения вихревых стенок в паре магнитостатически взаимодействующих нанолент под действием переменного магнитного поля, приложенного в плоскости лент. Кроме того, обсуждаем влияние постоянного магнитного поля, включенного перпендикулярно плоскости лент.

Формализм описания поведения магнитных вихрей как топологических неоднородностей в переменных полях достаточно хорошо развит (см. например [23-30] и библиографию). В основе аналитических расчетов лежит идеология представления уравнения Ландау-Лифшица через коллективные переменные [30-32]. В роли таких переменных выступают скорость и координаты центра магнитного вихря - ядра. Ядро - область с сильно неоднородной намагниченностью, направленной перпендикулярно поверхности магнетика. Ядро ведет себя в магнитном поле как квазичастица. Образование ядра результат конкуренции энергии обмена и магнитостатической энергии.

Состояние намагниченности вихря традиционно задается двумя параметрами: полярностью ядра $p= \pm 1$ и киральностью $q= \pm 1$. Знак полярности задается услов- 


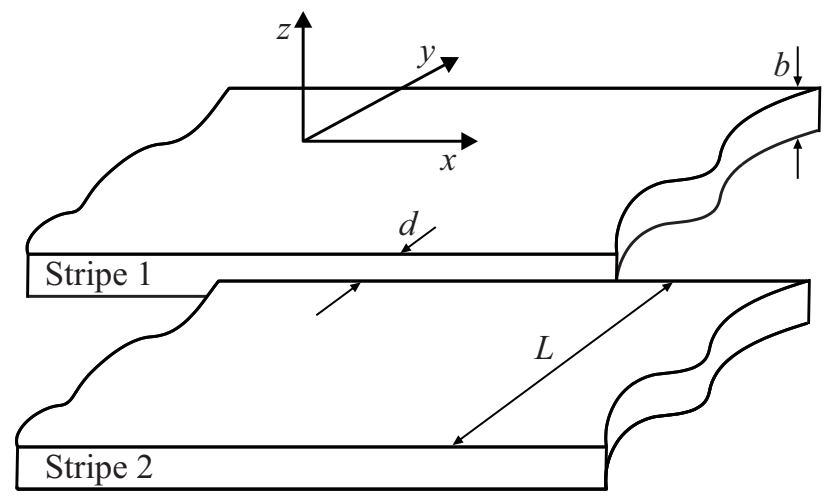

Рис. 1. Модель пары параллельно расположенных лент.

но: вдоль или против нормали к поверхности ленты. Знак киральности так же условен: вращение намагниченности по или против часовой стрелки. Часто для задания магнитного состояния вихрей удобно использовать топологический заряд $\pi_{T}=p q$. Характер движения ядра в наномагнетиках таков, как будто ядроквазичастица испытывает действие гироскопической силы $\mathbf{F}_{G}=\mathbf{G} \times \mathbf{v}$ [30]. Здесь $\mathbf{G}-$ гирровектор, $\mathbf{v}-$ скорость ядра. Величина гирровектора определяется выражением $|\mathbf{G}|=\pi_{T}\left(2 \pi M_{S} b / \gamma\right)(1-p h)$, где $b-$ толщина магнетика, $\gamma$ - гирромагнитное отношение, $M_{S}-$ намагниченность насыщения, $h=H /\left(\mu_{0} M_{S}\right)$ - безразмерное поле, приложенное перпендикулярно плоскости магнетика (вдоль или против намагниченности в центре ядра) $[26,32]$. Вихревая стенка в проволоках и лентах так же испытывает действие этой силы.

\section{2. Эффективная энергия вихревой доменной стенки в ленте}

Для получения решения уравнения движения намагниченности необходимо иметь функциональную зависимость потенциальной энергии магнитной подсистемы лент от обобщенных параметров. Получить строгий аналитический вид зависимости энергии от координат ядра вихря затруднительно. В лучшем случае для подобных систем удается приближенно представить энергию взаимодействующих вихревых стенок как систему взаимодействующих диполей, квадруполей. В этом приближении энергия выражается через сложные интегралы (см. например [12]), но вблизи равновесия энергия квадратична по координатам стенок [16]. Часто для получения практически значимых результатов используется компьютерное моделирование [6,16,17,33-35].

Выпишем далее эмпирическое выражение для потенциальной энергии, как функцию координаты ядра $W_{M}(\mathbf{r})$. Рассмотрим модель, состоящую из двух параллельно расположенных ферромагнитных лент толщиной $b$, шириной $L(b \ll L)$. Расстояние между лентами $d$. Длины лент много больше их ширины и толщины. Используемая система координат и модель показаны на рис. 1. Распределение намагниченности в нанолентах при наличии доменной структуры является результатом конкуренции нескольких видов энергий: обмена, размагничивающей, анизотропии и пр. В общем случае доменная стенка может включать в себя участки с традиционным разворотом намагниченности типа Нееля и вихревой участок [36,37].

Пример характерного распределения намагниченности показан на рис. 2. На этом рисунке длины стре-

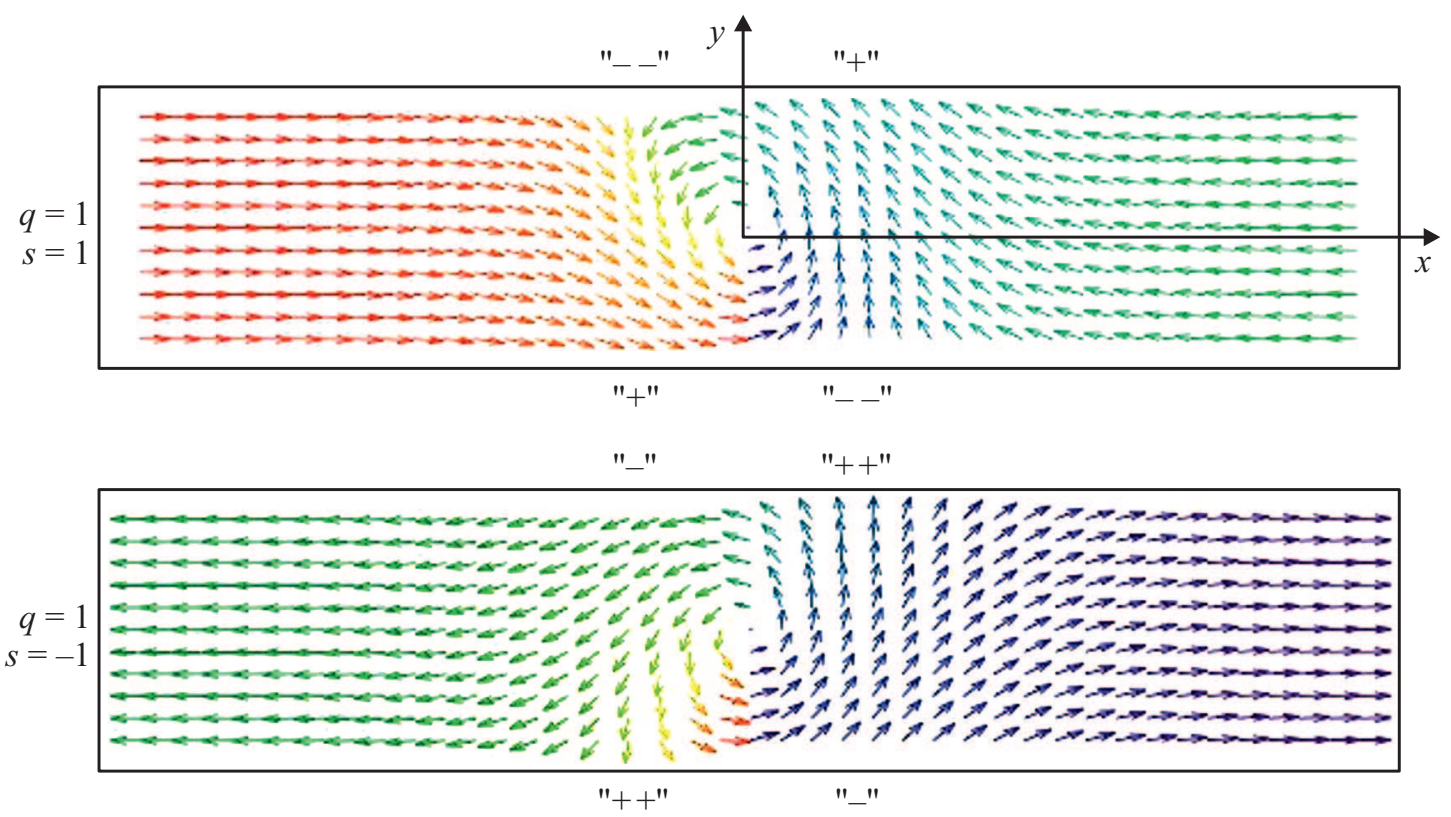

Рис. 2. Пример распределения намагниченности в вихревой стенке при разных сочетаниях параметров $q$ и $s$. Распределения построены для случая $\delta_{w}=L, \delta_{c}=0.2 \delta_{w}$. 
лок пропорциональны проекции единичного вектора намагниченности на плоскость $x y$. Параметр $s= \pm 1$ условно обозначает конфигурацию намамагниченности соседних доменов - направления к вихрю или от вихря. Заметим, что представление о конфигурации магнитного поля от магнитостатического заряда на боковой поверхности лент как от квадруполя (составных систем заря/диполь/квадруполь) не вполне корректно. Область локализации заряда на боковых поверхностях довольно велика (сравнима с шириной ленты $L$ ). Видно, что поверхностная плотность заряда $\sigma$ на боковой поверхности заметно отличается по модулю на участках с положительным и отрицательным знаками. Участки с большей плотностью на рис. 2 отмечены двойными знаками „++““ или „--“, а участки с меньшей плотностью одиночными знаками „++“ или „-““. Наблюдения распределения полей в реальных проволоках так же демонстрируют это свойство [13].

Механизм возникновения консервативных сил, действующих на ядро, следующий. При смещении ядра вихря меняется распределение плотности поверхностного заряда, на боковых поверхностях лент. Это приводит к изменению собственной энергии лент и их магнитостатического взаимодействия $W_{M}$. Следовательно, эффективные силы, которые испытывают ядра вихрей лент 1 и 2, как квазичастицы, могут быть записаны в виде: $\mathbf{F}_{\alpha}\left(\mathbf{r}_{1}, \mathbf{r}_{2}\right)=-\operatorname{grad}_{\alpha}\left(W_{M}\left(\mathbf{r}_{1}, \mathbf{r}_{2}\right)\right)$ (индекс $\alpha-$ номер ленты $\mathbf{r}_{1}, \mathbf{r}_{2}$ - радиус-вектора центров вихрей). Для оценочных расчетов хорошо себя зарекомендовала модель ,жесткого“ вихря. Ее суть состоит в пренебрежении изменением профиля распределения намагниченности при незначительном изменении координаты ядра [38-44]. В рамках этой модели для энергии магнитной подсистемы пары лент получим

$$
\begin{aligned}
W_{M}\left(x_{1}, y_{1}, x_{2}, y_{2}\right)= & W_{1_{\text {self }}}\left(x_{1}, y_{1}\right)+W_{2_{\text {self }}}\left(x_{2}, y_{2}\right) \\
& +W_{\text {int }}\left(x_{1}, y_{1}, x_{2}, y_{2}\right) .
\end{aligned}
$$

В правой части выражения (1) первое слагаемое $W_{1_{\text {self }}}\left(x_{1}, y_{1}\right)$ описывает собственную энергию первой ленты. Величина $W_{2_{\text {self }}}\left(x_{2}, y_{2}\right)$ - собственная энергия второй ленты, $W_{\text {int }}\left(x_{1}, y_{1}, x_{2}, y_{2}\right)$ - слагаемое, описывающее взаимодействие между магнитными подсистемами лент. В случае малых смещений вихрей из равновесного положения полную энергию можно представить в виде

$$
\begin{aligned}
W_{M}\left(x_{1}, y_{1}, x_{2}, y_{2}\right)= & \frac{1}{2} \chi\left(y_{1}-y_{0_{1}}\right)^{2}+\frac{1}{2} \chi\left(y_{2}-y_{0_{2}}\right)^{2} \\
& +w_{0} f_{x}(\Delta x) f_{y}(\Delta y) .
\end{aligned}
$$

Здесь $\chi-$ жесткость магнитной подсистемы изолированных лент, $\Delta x=x_{2}-x_{1}-\left(x_{0_{2}}-x_{0_{2}}\right), \Delta y=y_{2}-y_{2}$ $-\left(y_{0_{2}}-y_{0_{1}}\right), y_{1}, y_{2}, x_{1}, x_{2}$ - координаты ядер вихрей, $y_{0_{1}}, y_{0_{2}}, x_{0_{1}}, x_{0_{2}}-$ координаты ядер вихрей в равновесном положении, $w_{0}$ - некоторая постоянная, определяемая магнитными характеристиками системы.
Функции $f(\Delta x)$ и $f(\Delta y)$ должны монотонно убывать при увеличении модулей $|\Delta x|$ и $|\Delta y|$ соответственно.

Такой вид выражения для энергии системы выбран по следующим причинам. Энергия магнитостатического взаимодействия зарядов на боковых поверхностях изолированной ленты зависит только от координаты $y$, так как распределение заряда (следовательно, и энергия) меняется только при смещении ядра в поперечном направлении. Третье слагаемое отвечает за энергию взаимодействия зарядов на поверхностях, принадлежащих разным лентам. Эта энергия зависит от разности координат центров вихрей, как по оси $y$, так и по оси $x$. Важно заметить, что эффективная жесткость связи ядер вдоль оси $y$ зависит от расстояния между ними по оси $x$ и наоборот. Поэтому функции $f$ должны быть убывающими и зависеть от аргументов $\Delta x$ и $\Delta y$ в четных степенях. При малых смещениях функции $f$ могут быть разложены в ряд по степеням аргументов и с точностью до второго порядка представлены в виде

$$
f_{x}(\Delta x) \approx\left(1-\frac{\kappa_{x} \Delta x^{2}}{2 w_{0}}\right), f_{y}(\Delta y) \approx\left(1-\frac{\kappa_{y} \Delta y^{2}}{2 w_{0}}\right) .
$$

Здесь $\kappa_{x}, \kappa_{y}-$ жесткости связи ядер при смещении вдоль оси $x$ и $y$ соответственно.

Частный случай выражения (2) для невзаимодействующих лент удовлетворительно согласуется с результатами в $[6,15,16,45]$. Для дальнейших расчетов в предположении малых смещений ядер будем использовать это выражение.

\section{3. Циклическое движение вихревой стенки}

Рассмотрим далее поведение ядра вихря в переменном магнитном поле, приложенном в плоскости лент. Для описания динамического поведения магнитных вихрей продуктивным является уравнение Тиля [31]. Система уравнений Тиля применительно к вихревым стенкам двух лент имеет вид

$$
\left\{\begin{array}{l}
\mathbf{G}_{1} \times \mathbf{v}_{1}-D \mathbf{v}_{1}-\nabla W_{1}=0 \\
\mathbf{G}_{2} \times \mathbf{v}_{2}-D \mathbf{v}_{2}-\nabla W_{2}=0
\end{array}\right.
$$

Здесь $\mathbf{G}_{\alpha}=\pi_{T_{\alpha}} G_{0}\left(1-p_{\alpha} h\right) \mathbf{k}-$ гирровектор $(\mathbf{k}-$ единичный вектор вдоль оси $\left.\mathrm{z}, G_{0}=2 \pi M_{S} b / \gamma\right), \mathbf{v}-$ скорость ядра, $M_{S}-$ намагниченность насыщения, $\gamma$ - гиромагнитное отношение, $b$ - толщина лент, $D-$ коэффициент эффективного вязкого трения. Индексы обозначают принадлежность вихря к первой или второй ленте. Третье слагаемое в левой части уравнения (4) отвечает за консервативные силы, действующие на ядро вихря, как на квазичастицу. К таким силам отнесем возвращающую силу, силу взаимодействия с ядром вихря соседней ленты, эффективную силу, действующую на ядро вследствие взаимодействия намагниченности вихря с внешним магнитном полем. Таким образом: 
$W_{\alpha}=W_{M}\left(\mathbf{r}_{1}, \mathbf{r}_{2}\right)+W_{\alpha_{H}}$. Здесь $W_{\alpha_{H}}-$ энергия Зеемана вихревой доменной стенки в ленте с номером $\alpha$.

Тогда для обобщенных сил с учетом (2), действующих на ядра вихрей можно записать (i и $\mathbf{j}-$ единичные вектора вдоль осей $x$ и $y$ соответственно)

$$
\mathbf{F}_{\alpha}\left(\mathbf{r}_{1}, \mathbf{r}_{2}\right)=-\nabla W_{\alpha}=-\kappa_{x} \Delta x \mathbf{i}-\kappa_{y} \Delta y \mathbf{j}-\chi y_{\alpha} \mathbf{j}+\mathbf{F}_{\alpha_{H}} .
$$

Сила $\mathbf{F}_{\alpha_{H}}$, действующая на ядро из-за наличия переменного магнитного поля перпендикулярна направлению этого поля и лежит в плоскости ленты. Конкретное направление этой силы определяется киральностью вихря $q_{\alpha}[46,47]$. Заметим, что от величины постоянного магнитного поля, включенного перпендикулярно плоскости лент, зависят значения параметров $\mathbf{G}_{\alpha}, \kappa_{x}, \kappa_{y}, \chi$.

В проекции на систему координат (локальную для каждой ленты) система (4) принимает вид

$$
\left\{\begin{array}{l}
-G_{1} v_{y_{1}}-D v_{x_{1}}-\kappa_{x}\left(x_{1}-x_{2}\right)=-F_{1_{x}}, \\
G_{1} v_{x_{1}}-D v_{y_{1}}-\kappa_{y}\left(y_{1}-y_{2}\right)-\chi y_{1}=-F_{1_{y}}, \\
-G_{2} v_{y_{2}}-D v_{x_{2}}-\kappa_{x}\left(x_{2}-x_{1}\right)=-F_{2_{x}}, \\
G_{2} v_{x_{2}}-D v_{y_{2}}-\kappa_{y}\left(y_{2}-y_{1}\right)-\chi y_{2}=-F_{2_{y}} .
\end{array}\right.
$$

Здесь $F_{\alpha_{x}}, F_{\alpha_{y}}-x$ и $y$ проекции сил $\mathbf{F}_{\alpha_{H}}$, действующих на ядра вихрей. Пусть переменное поле меняется по закону: $\mathbf{F}_{\alpha_{H}}=q_{\alpha} \mathbf{F}_{0}(\exp (i \omega t)+\exp (-i \omega t)), \omega-$ циклическая частота изменения поля, $\mathbf{F}_{0}-$ амплитудное значение поля.

Пробные решения системы (6) выберем в виде

$$
\left\{\begin{array}{l}
x_{\alpha}=x_{0_{\alpha}}\left(\exp \left(i \pi_{T_{\alpha}} \omega t+\phi_{\alpha_{x}}\right)+\exp \left(-i \pi_{T_{\alpha}} \omega t-\phi_{\alpha_{x}}\right)\right), \\
y_{\alpha}=i y_{0_{\alpha}}\left(\exp \left(i \pi_{T_{\alpha}} \omega t+\phi_{\alpha_{y}}\right)-\exp \left(-i \pi_{T_{\alpha}} \omega t-\phi_{\alpha_{y}}\right)\right) .
\end{array}\right.
$$

Здесь $i-$ мнимая единица, $\phi_{\alpha_{x, y}}-$ разница фаз между законом изменения силы $\mathbf{F}_{\alpha_{H}}$ и законами изменения координат $x$ и $y$ соответственно. Тогда, после подстановки пробных решений в (6) для установившегося режима получим

$$
\left\{\begin{array}{r}
G_{0}\left(1-p_{1} h\right) \omega y_{0_{1}}-\left(\kappa_{x}+i D \omega\right) x_{0_{1}}-\kappa_{x} x_{0_{2}}=F_{0_{x}} q_{1}, \\
G_{0}\left(1-p_{1} h\right) \omega x_{0_{1}}-\left(\kappa_{y}+\chi+i D \omega\right) y_{0_{1}} \\
\quad-\pi_{T_{1}} \pi_{T_{2}} \kappa_{y} y_{0_{2}}=-i \pi_{T_{1}} F_{0_{y}} q_{1}, \\
G_{0}\left(1-p_{2} h\right) \omega y_{0_{2}}-\left(\kappa_{x}+i D \omega\right) x_{0_{2}}-\kappa_{x} x_{0_{1}}=F_{0_{x}} q_{2}, \\
G_{0}\left(1-p_{2} h\right) \omega x_{0_{2}}-\left(\kappa_{y}+\chi+i D \omega\right) y_{0_{2}} \\
-\pi_{T_{1}} \pi_{T_{2}} \kappa_{y} y_{0_{1}}=-i \pi_{T_{2}} F_{0_{y}} q_{2} .
\end{array}\right.
$$

Решение этой системы позволяет определить комплексные амплитуды

$$
\begin{aligned}
& x_{0_{1}}=-\frac{C_{x}}{G_{0}\left(1-p_{1} h\right)^{3}\left(1-p_{2} h\right)^{3} Z}, \\
& y_{0_{1}}=-\frac{C_{y}}{G_{0}\left(1-p_{1} h\right)^{3}\left(1-p_{2} h\right)^{3} Z} .
\end{aligned}
$$

Здесь введены обозначения:

$$
\begin{aligned}
& C_{x}=\left[F_{0_{x}}\left(q_{1}\left(\omega_{y}+\Omega+i \omega \omega_{\Gamma}\right)\left(1-p_{2} h\right)-p_{2} \pi_{T_{1}} \omega_{y}\left(1-p_{1} h\right)\right)\right. \\
& \left.-i F_{0_{y}} p_{1} \omega\left(1-p_{1} h\right)\left(1-p_{2} h\right)\right]\left[\left(\omega_{x}+i \omega \omega_{\Gamma}\right)\left(\omega_{y}+\Omega+i \omega \omega_{\Gamma}\right)\right. \\
& \left.\times\left(1-p_{1} h\right)+\pi_{T_{1}} \pi_{T_{2}} \omega_{x} \omega_{y}\left(1-p_{2} h\right)-\omega^{2}\left(1-p_{1} h\right)\left(1-p_{2} h\right)^{2}\right] \\
& +\left[F_{0_{x}}\left(q_{2}\left(\omega_{y}+\Omega+i \omega \omega_{\Gamma}\right)\left(1-p_{1} h\right)-p_{1} \pi_{T_{2}} \omega_{y}\left(1-p_{2} h\right)\right)\right. \\
& \left.-i F_{0_{y}} p_{2} \omega\left(1-p_{1} h\right)\left(1-p_{2} h\right)\right]\left[\omega_{x}\left(\omega_{y}+\Omega+i \omega \omega_{\Gamma}\right)\left(1-p_{2} h\right)\right. \\
& \left.+\pi_{T_{1}} \pi_{T_{2}}\left(\omega_{x}+i \omega \omega_{\Gamma}\right) \omega_{y}\left(1-p_{1} h\right)\right] \\
& C_{y}=\left[F_{0_{x}}\left(q_{1}\left(\omega_{y}+\Omega+i \omega \omega_{\Gamma}\right)\left(1-p_{2} h\right)-p_{2} \pi_{T_{1}} \omega_{y}\left(1-p_{1} h\right)\right)\right. \\
& \left.-i F_{0_{y}} p_{1} \omega\left(1-p_{1} h\right)\left(1-p_{2} h\right)\right]\left[\omega_{x}\left(\omega_{y}+\Omega+i \omega \omega_{\Gamma}\right)\left(1-p_{1} h\right)\right. \\
& \left.+\pi_{T_{1}} \pi_{T_{2}} \omega_{y}\left(\omega_{x}+i \omega \omega_{\Gamma}\right)\left(1-p_{2} h\right)\right]+\left[F _ { 0 _ { x } } \left(q_{2}\left(\omega_{y}+\Omega+i \omega \omega_{\Gamma}\right)\right.\right. \\
& \left.\times\left(1-p_{1} h\right)-p_{1} \pi_{T_{2}} \omega_{y}\left(1-p_{2} h\right)\right)-i F_{0_{y}} p_{2} \omega\left(1-p_{1} h\right) \\
& \left.\times\left(1-p_{2} h\right)\right]\left[\left(\omega_{x}+i \omega \omega_{\Gamma}\right)\left(\omega_{y}+\Omega+i \omega \omega_{\Gamma}\right)\left(1-p_{2} h\right)\right. \\
& \left.+\pi_{T_{1}} \pi_{T_{2}} \omega_{x} \omega_{y}\left(1-p_{1} h\right)-\omega^{2}\left(1-p_{1} h\right)^{2}\left(1-p_{2} h\right)\right], \\
& Z=\omega^{4}-\omega^{2}\left[\left(\omega_{x}+i \omega \omega_{\Gamma}\right)\left(\omega_{y}+\Omega+i \omega \omega_{\Gamma}\right)\right. \\
& \left.\times\left(\frac{1}{\left(1-p_{1} h\right)^{2}}+\frac{1}{\left(1-p_{2} h\right)^{2}}\right)+\frac{2 \pi_{T_{1}} \pi_{T_{2}} \omega_{x} \omega_{y}}{\left(1-p_{1} h\right)\left(1-p_{2} h\right)}\right] \\
& +\frac{\left[\left(\omega_{x}+i \omega \omega_{\Gamma}\right)^{2}-\omega_{x}^{2}\right]\left[\left(\omega_{y}+\Omega+i \omega \omega_{\Gamma}\right)^{2}-\omega_{y}^{2}\right]}{\left(1-p_{1} h\right)^{2}\left(1-p_{2} h\right)^{2}},
\end{aligned}
$$

где $\omega_{\Gamma}=D / G_{0}-$ безразмерная величина, $\Omega=\chi / G_{0}$, $\omega_{x}=\kappa_{x} / G_{0}, \omega_{y}=\kappa_{y} / G_{0}$.

Разность фаз между законами изменения вынуждающей силы и законами движения ядер вихрей определяется из выражений:

$$
\left\{\begin{array} { l } 
{ \operatorname { s i n } ( \phi _ { \alpha _ { x } } ) = \frac { \operatorname { I m } ( x _ { 0 _ { \alpha } } ) } { | x _ { 0 _ { \alpha } } | } , } \\
{ \operatorname { c o s } ( \phi _ { \alpha _ { x } } ) = \frac { \operatorname { R e } ( x _ { 0 _ { \alpha } } ) } { | x _ { 0 _ { \alpha } } | } . }
\end{array} \quad \left\{\begin{array}{l}
\sin \left(\phi_{\alpha_{y}}\right)=\pi_{T_{\alpha}} \frac{\operatorname{Im}\left(y_{0_{\alpha}}\right)}{\left|y_{0_{\alpha}}\right|}, \\
\cos \left(\phi_{\alpha_{y}}\right)=\pi_{T_{\alpha}} \frac{\operatorname{Re}\left(y_{0_{\alpha}}\right)}{\left|y_{0_{\alpha}}\right|} .
\end{array}\right.\right.
$$

Множитель $\pi_{T_{\alpha}}$ необходим для учета направления вращения ядра.

В частном случае пренебрежимо малого затухания $\left(\omega_{\Gamma}=0\right)$ собственные частоты мод легко определить из 
Набор собственных частот системы $\omega_{s}$, рассчитанных из (14), для различных сочетаний полярностей и киральностей вихревых стенок

\begin{tabular}{c|c|c}
\hline № & Сочетание $p_{\alpha}$ и $q_{\alpha}\left\{p_{1}, p_{2}, q_{1}, q_{2}\right\}$ & Частота коллективной моды $\omega_{s}$ \\
\hline 1 & $\{1,1, \pm 1, \pm 1\}$ & $\frac{2 \omega_{x}\left(\Omega+2 \omega_{y}\right)}{(1-h)^{2}}$ \\
\hline 2 & $\{1,1, \pm 1, \mp 1\}$ & $\frac{2 \omega_{x} \Omega}{(1-h)^{2}}$ \\
\hline 3 & $\{ \pm 1, \mp 1,1,1\},\{ \pm 1, \mp 1,-1,-1\}$ & $\frac{2 \omega_{x}}{\left(1-h^{2}\right)^{2}}\left(\Omega\left(1+h^{2}\right)+2 \omega_{y} h^{2}\right)$ \\
\hline 4 & $\{ \pm 1, \mp 1,1,-1\},\{ \pm 1, \mp 1,-1,1\}$ & $\frac{2 \omega_{x}}{\left(1-h^{2}\right)^{2}}\left(\Omega\left(1+h^{2}\right)+2 \omega_{y}\right)$ \\
\hline 5 & $\{-1,-1, \pm 1, \pm 1\}$ & $\frac{2 \omega_{x}\left(\Omega+2 \omega_{y}\right)}{(1+h)^{2}}$ \\
\hline 6 & $\{-1,-1, \pm 1, \mp 1\}$ & $\frac{2 \omega_{x} \Omega}{(1+h)^{2}}$
\end{tabular}
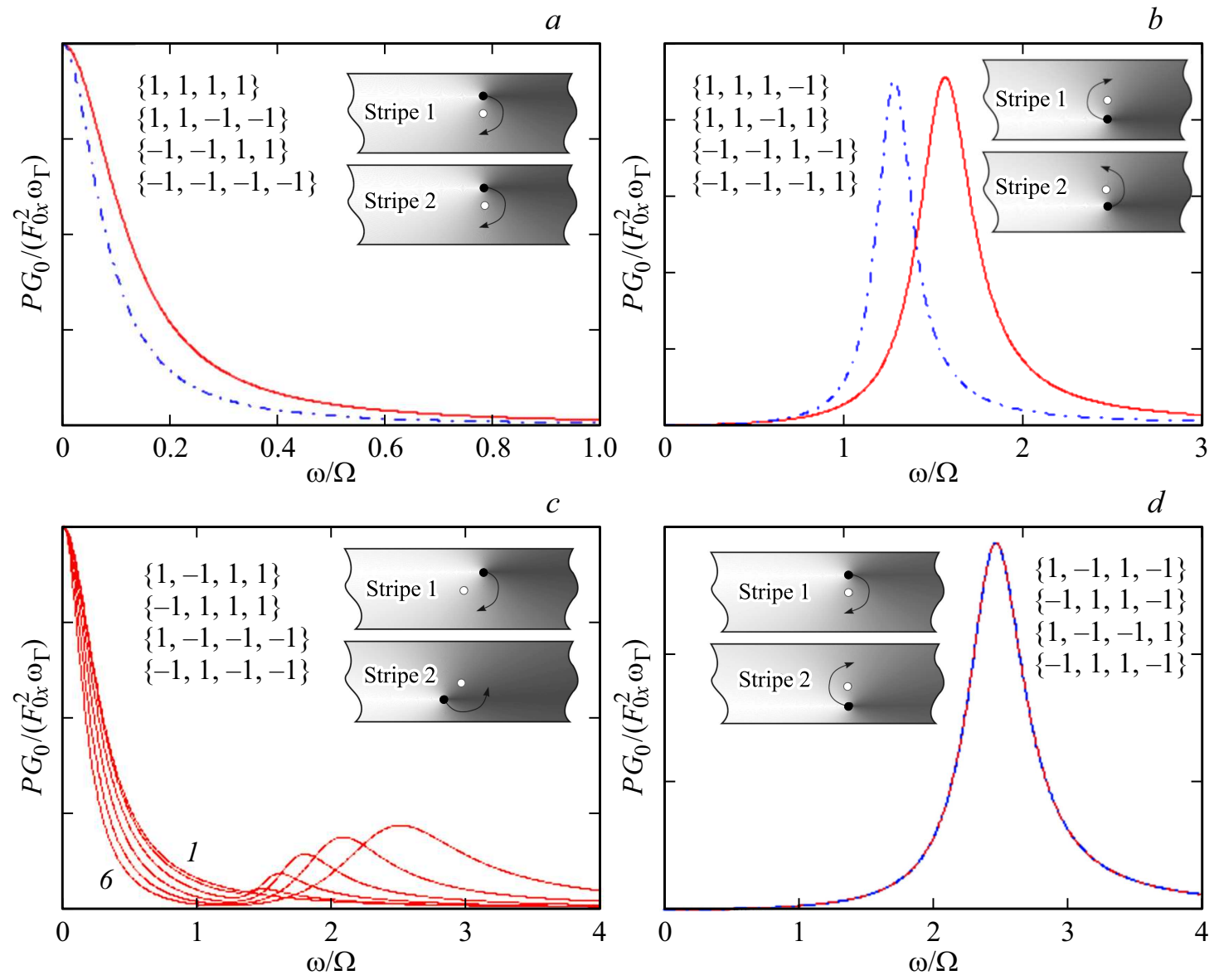

Рис. 3. Зависимость поглощаемой мощности (измеряется в безразмерных условных единицах) от частоты переменного поля. Переменное поле приложено вдоль оси $y$. Кривые построены для параметров: $\omega_{y}=\Omega, \omega_{x}=\omega_{y}, \omega_{\Gamma}=0.1, h=0.1$. $a, b-$ штрихпунктирными кривыми показана мощность для случаев, когда постоянное поле приложено против оси $z ; c, d-$ случаи постоянного поля, приложенного вдоль $z$ и против $z$ совпадают; на $c$ - показано семейство кривых $1-6$ при постоянных полях $h=0 \ldots .5 \mathrm{c}$ шагом $\Delta h=0.1$. На вставках в фигурных скобках показаны сочетания полярностей и киральностей вихревых стенок $\left\{p_{1}, p_{2}, q_{1}, q_{2}\right\}$ и изображены фазы и направления движения ядер вихрей. Черной точкой показаны ядра, светлыми - положения начала координат. 

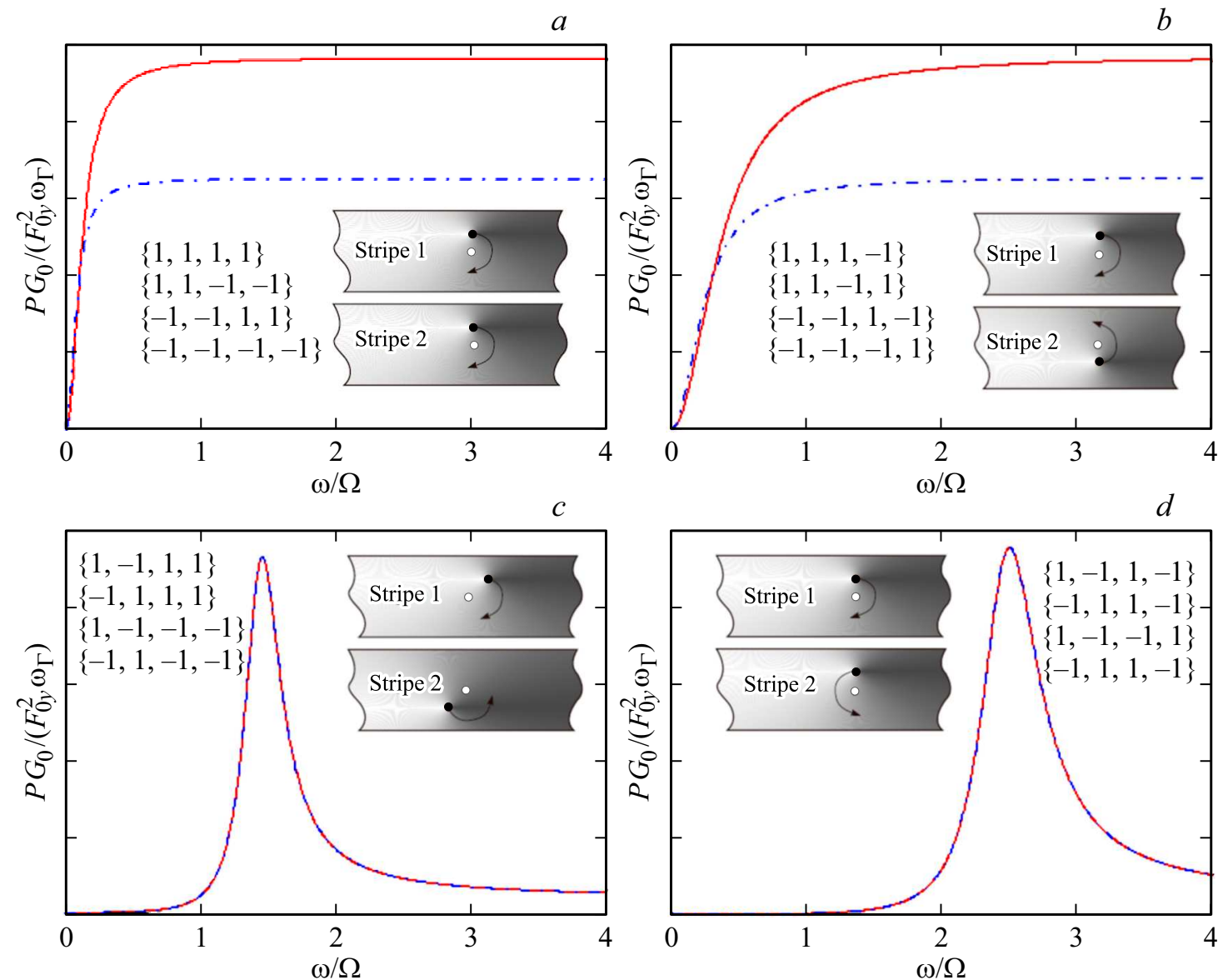

Рис. 4. Зависимость поглощаемой мощности (в условных единицах) от частоты переменного поля, приложенного вдоль оси $x$. Кривые построены для тех же параметров, что на рис. 3.

условия $|Z|=0$. В результате получим

$$
\begin{aligned}
\omega_{s}^{2}= & \omega_{x} \Omega\left(\frac{1}{\left(1-p_{1} h\right)^{2}}+\frac{1}{\left(1-p_{2} h\right)^{2}}\right) \\
& +\omega_{x} \omega_{y}\left(\frac{\pi_{T_{1}}}{1-p_{1} h}+\frac{\pi_{T_{2}}}{1-p_{2} h}\right)^{2} .
\end{aligned}
$$

Распределение полярностей, киральностей и значения частот приведены в таблице. Вследствие того, что обе ленты, образующие пару одинаковы по размеру и магнитным характеристикам, величины $G_{0}, D, \chi$ лент одинаковы. Это приводит к вырождению состояний по частоте. В частности, каждое из состояний под номерами 1, 2, 5, 6 из таблицы вырождены двукратно, а состояния 3 и 4 четырехкратно. В результате мы имеем шесть собственных частот.

В отсутствие постоянного перпендикулярного поля $(h=0)$ из выражения (14) для резонансных частот получим получим

$$
\omega_{s}=\sqrt{2 \omega_{x} \Omega+2 \omega_{x} \omega_{y}\left(1+\pi_{T_{1}} \pi_{T_{2}}\right)} .
$$

Состояния с определенными сочетаниями киральности, но противоположными полярностями при отсутствии поля $h$ не различаются. Следовательно, при $\omega_{\Gamma} \rightarrow 0$ степень вырождения усиливается. В результате из таблицы неразличимыми становятся наборы состояний $\{1,4,5\}$, и отдельно $\{2,3,6\}$. Заметим, что в отсутствие постоянного поля $h$ важно не различие полярностей и киральностей, а различие их произведения $p q$, т.е. топологических зарядов $\pi_{T_{\alpha}}$, что и отражено в формуле (15), из которой следует, что при $h=0$ имеются только две различающиеся частоты: $\omega_{s}=\sqrt{2 \omega_{x} \Omega}$ и $\omega_{s}=\sqrt{2 \omega_{x} \Omega+4 \omega_{x} \omega_{y}}$. В работе [48] теоретически рассмотрена динамика двух взаимодействующих вихрей внутри одной ленты. Авторами был получен результат для частоты коллективного движения вихрей, являющийся частным случаем формулы (15).

Поглощаемую энергию парой лент можно оценить по формуле

$$
P(\omega, h) \approx D \omega^{2}\left(\left|x_{0_{1}}\right|^{2}+\left|y_{0_{1}}\right|^{2}+\left|x_{0_{2}}\right|^{2}+\left|y_{0_{2}}\right|^{2}\right) .
$$

Зависимость поглощаемой мощности (безразмерной) от частоты внешнего переменного поля, включенного вдоль оси $y$, изображена на рис. 3, а вдоль оси $x-$ на рис. 4. Эти графики требуют комментариев.

Прежде всего отметим, что в рассматриваемой модели ленты длинные и вихревые стенки находятся на 
$a$

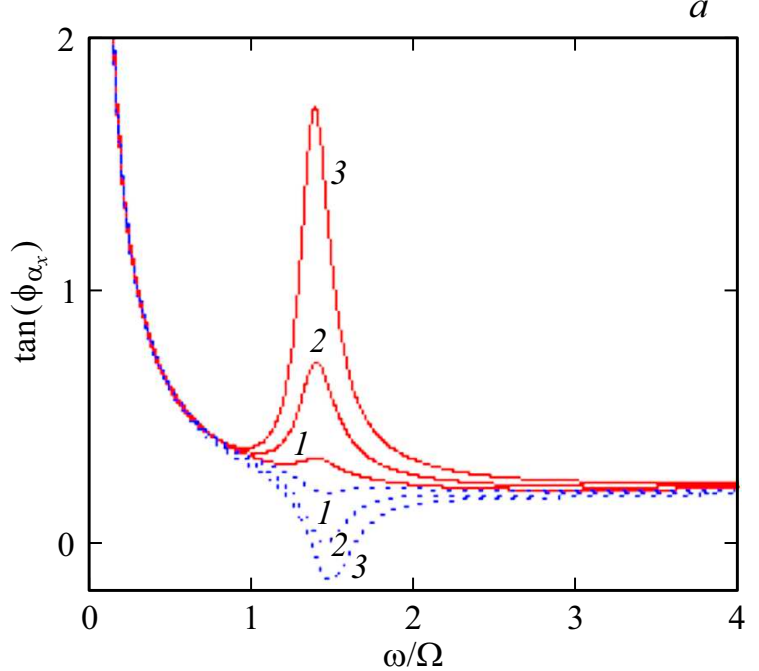

$b$

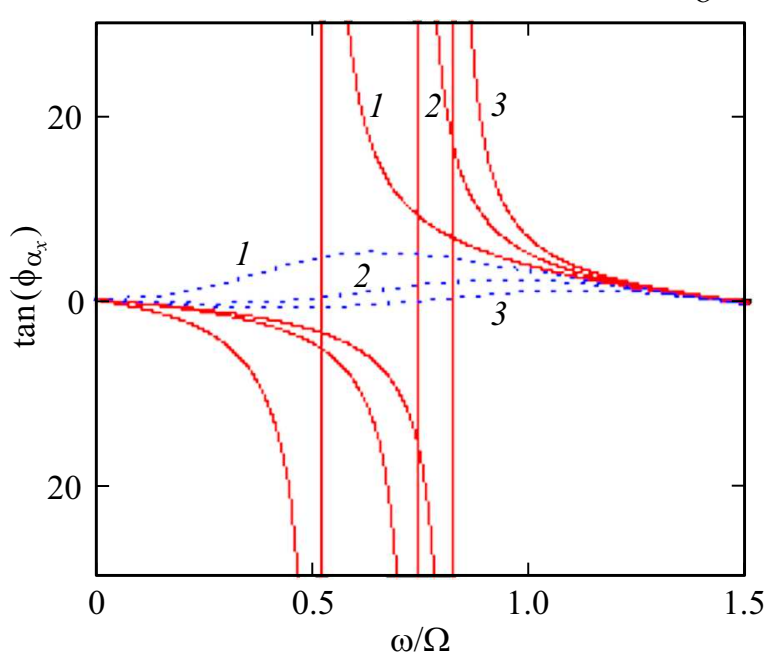

Рис. 5. Зависимость тангенса фазы $x$-координаты ядра магнитного вихря в ленте 1 (сплошные кривые) и ленте 2 (точки). Кривые $1-3$ построены для разных значений постоянного перпендикулярного поля: $h_{1}=0.01, h_{2}=0.05$ и $h_{3}=0.1$ соответственно. $a$ - график построен для случая, когда переменное поле приложено вдоль оси $y, b-$ для переменного поля, приложенного вдоль оси $x$.

значительном удалении от торцов. Поэтому магнитная энергия изолированной ленты не меняется при смещении центра вихря вдоль оси $x$. Следовательно, вдоль оси $x$ не возникает возвращающая сила. В изолированной ленте при смещении ядра вихря вдоль оси у энергия магнитной подсистемы меняется вследствие перераспределения магнитных зарядов на боковых поверхностях ленты. Поэтому вдоль оси $y$ возникает возвращающая сила независимо от присутствия вблизи второй ленты. Эта особенность накладывает отпечаток на характер движения стенки в переменном магнитном поле.

Состояние, показанное на рис. 3, $a$ не проявляет резонансных свойств. В зависимости от взаимной ориентации постоянного перпендикулярного поля $h$ и полярности ядра $p_{\alpha}$ мы имеем монотонно спадающие зависимости от частоты с разной скоростью. Расчет разности фаз движения ядер стенок в лентах 1 и 2 с помощью формул (13) показал, что вращение происходит синхронно $\left(\phi_{1_{x}}=\phi_{2_{x}}, \phi_{1_{y}}=\phi_{2_{y}}\right)$. При таких сочетаниях полярностей и киральностей вихрей взаимодействие между доменными стенками не проявляется, так как в любой момент времени выполняются условия: $\Delta x=0, \Delta y=0$. В этом случае в уравнениях движения отсутствует проекция возвращающей силы вдоль оси $x$ и система не является колебательной.

Аналогичный эффект можно наблюдать в случае переменного поля, включенного вдоль оси $y$ (см. рис. $4, a, b)$. Расчет фаз для этих сочетаний $\left\{p_{1}, p_{2}, q_{1}, q_{2}\right\}$ показал, что координаты $x_{1}$ и $x_{2}$ меняются со временем синхронно. Это означает отсутствие возвращающей силы вдоль оси $x$.

Иначе ведет себя система в случае, если координаты центров вихрей $x_{1}$ и $x_{2}$ меняются со временем не синхронно. В этом случае у обобщенной силы взаимо- действия между ядрами имеется ненулевая проекция на ось $x$, т. е. существует возвращающий фактор не только вдоль оси $y$, но и вдоль оси $x$. При этом система имеет признаки колебательной и в переменном поле существуют резонансные состояния. Такие сочетания $\left\{p_{1}, p_{2}, q_{1}, q_{2}\right\}$ показаны на рис. $3, b, c, d, 4, c, d$. На вставках в этих графиках изображены направления и фазы движения ядер вихрей в некоторые моменты времени.

Интересными являются состояния с одинаковыми киральностями, но противоположными полярностями (рис. 3, с и 4,c). В случае, когда переменное поле приложено вдоль оси $y$ резонансный пик проявляется только при наличии постоянного поля, перпендикулярного плоскости лент. При переменном поле, приложенном вдоль оси $x$ резонанс реализуется при любых значениях перпендикулярного поля $h$. В этих состояния сдвиг фаз между положениями центров вихрей лент $\phi_{1_{x, y}}-\phi_{2_{x, y}}$ зависит от частоты переменного поля $\omega$ и величины постоянного поля $h$. Этой зависимости нет при остальных сочетаниях $\left\{p_{1}, p_{2}, q_{1}, q_{2}\right\}$, где реализуются только две возможности: $\phi_{1_{x, y}}-\phi_{2_{x, y}}=0, \pi$. Поведение фаз вращения ядер лент для обсуждаемого случая показано на рис. 5 .

\section{4. Заключение}

Анализ и решение уравнений движения вихрей показал, что существуют определенные сочетания полярностей и киральностей вихревых стенок, при которых их движение носит периодический характер. В пренебрежении затуханием получено простое выражение для частот резонансов в зависимости от топологического заряда вихрей, величины и направления постоянного магнит- 
ного поля, включенного перпендикулярно поверхности лент.

Знание состояний взаимодействующих вихревых стенок, при которых реализуется резонансное или нерезонансное поведение в переменных полях открывает возможности для целенаправленного управления намагниченностью в массивах параллельно расположенных лент. Это особенно важно в контексте разработки устройств хранения информации.

Зависимость частот мод коллективного движения вихревых стенок от внешнего поля позволяет считать подобные системы хорошими кандидатами для применения в разнообразных датчиках полей и прочих устройств спинтроники. Существование состояний, частота резонанса которых чувствительна к направлению перпендикулярного поля (рис. 3,b), открывает возможность конструирования датчиков, определяющих не только величину поля, но и его направление.

\section{Список литературы}

[1] D.A. Allwood, G. Xiong, C.C. Faulkner, D. Atkinson, D. Petit, R.P. Cowburn. Science, 309, 1688(2005).

[2] M. Hayashi, L. Thomas, R. Moriya, Ch. Rettner, S.S.P. Parkin. Science, 320209 (2008).

[3] S.S.P. Parkin, M. Hayashi, L. Thomas. Science 320, 190 (2008).

[4] S. Jamet, N. Rougemaille, J.C. Toussaint, O. Fruchart. arXiv:1412.0679v1 [cond-mat.mes-hall] 1 Dec 2014.

[5] A. Thiaville, Yo. Nakatani. Appl. Physics 101, 161 (2006).

[6] H. Youk, G.-W. Chern, K. Merit, B. Oppenheimer, O. Tchernyshyov. J. Appl. Phys. 99, 08B101 (2006).

[7] Yo. Nakatania, A. Thiavilleb, J. Miltat. JMMM 290-291, 750 (2005).

[8] V.D. Nguyen, O. Fruchart, S. Pizzini, J. Vogel, J.-C. Toussaint, N. Rougemaille. Sci. Rep. 5, 12417 (2015).

[9] N. Rougemaille, V. Uhlir, O. Fruchart, S. Pizzini, J. Vogel, J.C. Toussaint. Appl. Phys. Lett. 100, 172404 (2012).

[10] А.К. Звездин, В.И. Белотелов, К.А. Звездин. Письма в ЖЭТФ 87, 443 (2008).

[11] В.В. Волков, В.А. Боков. ФТТ 50, 193 (2008).

[12] A. Janutka. Acta Phys. Pol. A 124, 641 (2013).

[13] A.G. Kozlov, M.E. Stebliy, A.V. Ognev, A.S. Samardak, A.V. Davydenko, L.A. Chebotkevich. JMMM 422, 452 (2017).

[14] M. Shen, Yue Zhang, L. You, X. Yang. (https://arxiv.org/abs/1807.11063)

[15] D.J. Clarke, O.A. Tretiakov, G.-W. Chern, Ya.B. Bazaliy, O. Tchernyshyov. Phys. Rev. B 78, 134412 (2008).

[16] Y. Su, J. Sun, J. Hu, H. Lei. EPL 103, 67004 (2013).

[17] S.-K. Kim, J.-Y. Lee, Y.-S. Choi, K. Yu. Guslienko, K.-S. Lee. Appl. Phys. Lett. 93, 052503 (2008).

[18] J. Yang, C. Nistor, G.S.D. Beach, J.L. Erskine. Phys. Rev. B 77, 014413 (2008).

[19] K.Yu. Guslienko, J.-Y. Lee, S.-K. Kim. IEEE Tran. Magn. 44, 3079-3082 (2008).

[20] N. Locatelli, A.E. Ekomasov, A.V. Khvalkovskiy, Sh.A. Azamatov, K.A. Zvezdin, J. Grollier, E.G. Ekomasov, V. Cros. Appl. Phys. Lett. 102, 062401 (2013).
[21] I. Purnama, M. Chandra Sekhar, S. Goolaup, W.S. Lew. Appl. Phys. Lett. 99, 152501 (2011).

[22] Ch. Murapaka, S. Goolaup, I. Purnama, W.S. Lewa. Appl. Phys. Lett. 117, 053913 (2015).

[23] O. Klein, G. de Loubens, V.V. Naletov, F. Boust, T. Guillet, H. Hurdequint, A. Leksikov, A.N. Slavin, V.S. Tiberkevich, N. Vukadinovic. Phys. Rev. B 78, 144410 (2008)

[24] K.-S. Lee, S.-K. Kim. Appl. Phys. Lett. 91, 132511 (2007)

[25] B.A. Ivanov, G.M. Wysin. Phys. Rev. B 65, 134434 (2002)

[26] K.Yu. Guslienko, B.A. Ivanov, V. Novosad, Y. Otani, H. Shima, K. Fukamichi. J. Appl. Phys. 91, 8037 (2002)

[27] В.А. Орлов, Р.Ю. Руденко, А.В. Кобяков, А.В. Лукьяненко, П.Д. Ким, В.С. Прокопенко, И.Н. Орлова. ЖЭТФ 153, 635 (2018).

[28] F. Abreu Araujo, M. Darques, K.A. Zvezdin, A.V. Khvalkovskiy, N. Locatelli, K. Bouzehouane, V. Cros, L. Piraux. Phys. Rev. B 86, 064424 (2012).

[29] A.D. Belanovsky, N. Locatelli, P.N. Skirdkov, F. Abreu Araujo, J. Grollier, K.A. Zvezdin, V. Cros, A.K. Zvezdin. Phys. Rev. B 85, 100409(R) (2012).

[30] A.K. Zvezdin, K.A. Zvezdin. Bull. Lebedev Phys. Institute 37, 8, 240 (2010).

[31] A. Thiele. Phys. Rev. Lett. 30, 230 (1973).

[32] J. Kim, S.-B. Choe. J. Magn. 12, 3, 113 (2007).

[33] R.D. McMichael, M.J. Donahue. IEEE Trans. Magn. 33, 4167 (1997).

[34] O.A. Tretiakov, D. Clarke, Gia-Wei Chern, Ya.B. Bazaliy, O. Tchernyshyov. Phys. Rev. Lett. 100, 127204 (2008).

[35] М.Н. Дубовик, Б.Н. Филиппов, Л.Г. Корзунин. ФММ 117, 342 (2016).

[36] G.-W. Chern, H. Youk, O. Tchernyshyov. J. Appl. Phys. 99, 08Q505 (2006).

[37] J. He, Z. Li, S. Zhang. Phys. Rev. B 73, 184408 (2006).

[38] U.K. Rossler, A.N. Bogdanov, K.-H. Muller. IEEE Tran. Magn. 38, 2586 (2002).

[39] K.Yu. Guslienko, V. Novosad, Y. Otani, H. Shima, K. Fukamichi. Phys. Rev. B 65, 024414 (2001).

[40] V.P. Kravchuk, D.D. Sheka. Phys. Solid State 49, 1923 (2007).

[41] П.Д. Ким, В.А. Орлов, В.С. Прокопенко, С.С. Замай, В.Я. Принц, Р.Ю. Руденко, Т.В. Руденко. ФТТ 57, 29 (2015); [P.D. Kim, V.A. Orlov, V.S. Prokopenko, S.S. Zamai, V.Ya. Prints, R.Yu. Rudenko, T.V. Rudenko. Phys. Solid State 57, 30 (2015)].

[42] K.Yu. Guslienko, X.F. Han, D.J. Keavney, R. Divan, S.D. Bader. Phys. Rev. Lett. 96, 067205 (2006).

[43] M. Wolf, U.K. Robler, R. Schafer. JMMM 314, 105 (2007).

[44] П.Д. Ким, В.А. Орлов, Р.Ю. Руденко, В.С. Прокопенко, И.Н. Орлова, С.С. Замай. Письма в ЖЭТФ 101, 620 (2015).

[45] A. Janutka. Acta Phys. Pol. A 124, 23 (2013).

[46] S.-B. Choe, Y. Acremann, A. Scholl, A. Bauer, A. Doran, J. Stohr, H.A. Padmore. Science 304, 420 (2010).

[47] J.Y. Lee, K.S. Lee, S. Choi, K. Yu. Guslienko, S.-K. Kim. Phys. Rev B 76, 184408 (2007).

[48] Y. Talbi, Y. Roussigne, P. Djemia, M. Labrune. J. Phys.: Conf. Ser. 200, 042027 (2010).

Редактор Т.Н. Василевская 